\title{
On-line multichannel Raman spectroscopic detection system for capillary zone electrophoresis
}

\author{
CHANG-YUH CHEN and MICHAEL D. MORRIS* \\ Department of Chemistry, University of Michigan, Ann Arbor, MI 48109 (U.S.A.) \\ (Received August 28th, 1990)
}

\begin{abstract}
An on-line multichannel Raman spectroscopic detection system for capillary zone electrophoresis using a charge-coupled device as the detector is described. Resonant, near-resonant and non-resonant excitation Raman spectroscopies are employed. The $400 \mathrm{~cm}^{-1}$ spectral window provides adequate information to identify resolved and unresolved compounds. The use of analyte velocity reduction to allow increased data acquisition times is described. With near-resonant enhancement, the technique is shown to allow detection of 500 attomoles of methyl orange from a 5-nl injection volume, corresponding to $1 \cdot 10^{-7}$ $M$.
\end{abstract}

\section{INTRODUCTION}

Since its emergence in the early 1980's, capillary zone electrophoresis (CZE) has become widely accepted as a high resolution separation technique [1,2]. While ultraviolet absorption spectroscopy and fluorescence are useful, there remains a need for detection systems which provide better molecular characterization. But, the small internal diameters $(10-75 \mu \mathrm{m})$ of electrophoresis capillaries and the consequent small sample loadings have proven to be serious problems in development of high resolution spectroscopic detertors for capillary electrophoresis. Only electrospray ionization mass spectrometric detection [3] has yet proven practical.

In an earlier communication, we demonstrated the feasibility of resonance Raman detection with a single stage monochromator and a photomultiplier detection system [4]. Although resonance Raman spectroscopy was shown to have about the same detection limits as absorption spectroscopy, the single channel detector system was unable to exploit fully the fingerprinting capability of vibrational spectra. With a multichannel detector to allow fingerprinting Raman spectroscopy may be an attractive detection system for CZE.

Since the mid-1980's the slow-scan charge-coupled device (CCD) detector has emerged as the best general purpose multichannel Raman detector [5,6]. The CCD provides low read noise, nearly-zero dark current, high responsivity, multichannel capability and large dynamic range. This detector system appears well-suited to on-line Raman spectroscopic detection for CZE. 
Vo-Dinh and co-workers [7] have already reported an on-line CCD-based fluorescence detector for CZE. These authors have shown the evolution of a fluorescence spectrum at 6-s intervals during electrophoresis and have demonstrated that the CCD provides better signal-to-noise ratio than a photomultiplier tube (PMT) in on-line applications. In the present communication we demonstrate on-line CCD detection of Raman spectra over a $400-\mathrm{cm}^{-1}$ interval, using resonant, near-resonant and non-resonant excitation.

\section{EXPERIMENTAL}

\section{Capillary zone electrophoresis}

A block-diagram of the experiment is shown in Fig. 1. The CZE apparatus described previously [4] was used with only minor modification. In particular, the number of collection fibers was reduced from ten to two, and the capillary/fiber holder was redesigned, as shown in Fig. 2, to provide more rigid mounting of the capillary. A $55 \mathrm{~cm} \times 75 \mu \mathrm{m}$ I.D. capillary (Polymicro Technologies TSP/075/375) was used as separation capillary. The detection region was formed by burning off a small section of protective coating about $5 \mathrm{~cm}$ from the exit end of the capillary. Sample introduction was by electromigration $(5-10 \mathrm{~s}, 20 \mathrm{kV})$. The usual electrophoresis working voltage was $20 \mathrm{kV}$, at a current of $65-70 \mu \mathrm{A}$. Electrophoresis was carried out in a 0.02 $M$ Trizma buffer (Tris[hydroxymethyl]aminomethane) at $\mathrm{pH} 7.15$.

\section{Raman spectroscopic detector}

The on-line multichannel Raman spectroscopic detection system is shown in Fig. 1. The excitation source was a frequency doubled Nd-YAG laser (Quantronix 416), operated at $532 \mathrm{~nm}$. The laser power at the capillary was about $300 \mathrm{~mW}$. A $5 \times$

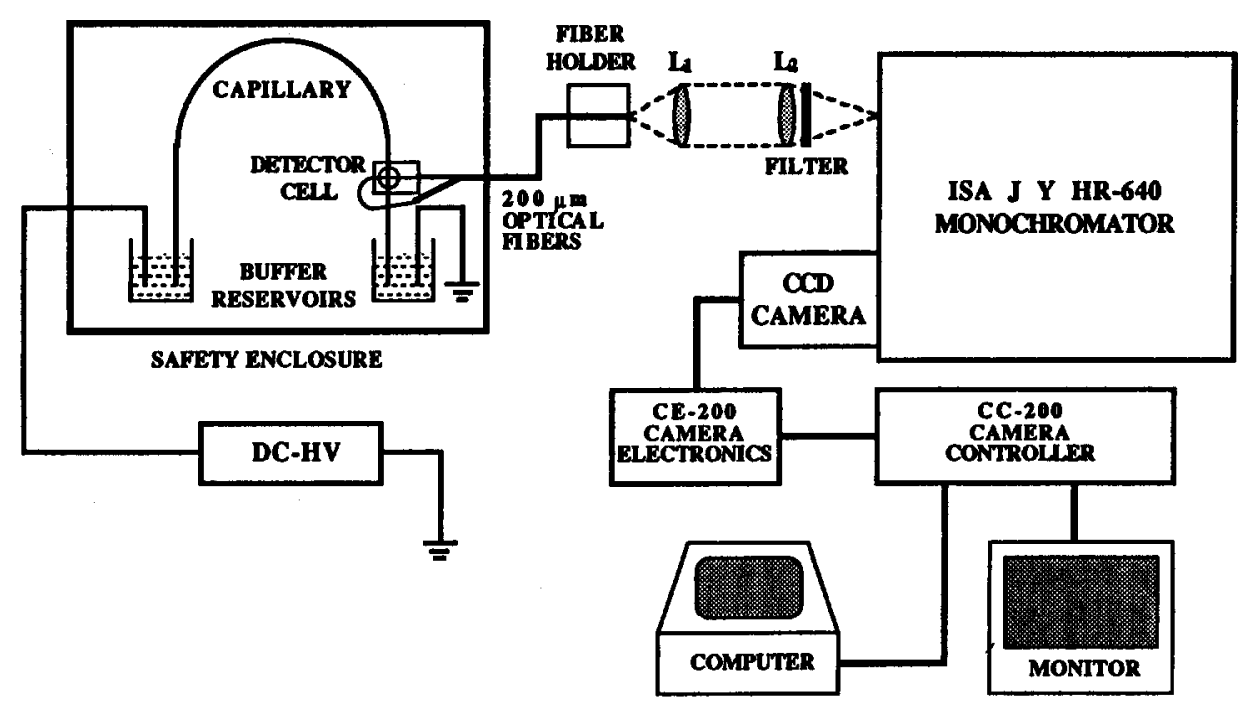

Fig. 1. Instrumentation for capillary zone electrophoresis with a CCD Raman spectroscopic detector. $\mathbf{L}_{1}$, $f=50 \mathrm{~mm} ; \mathrm{L}_{2}, f=200 \mathrm{~mm}$; Filter, Schott OG-550. 


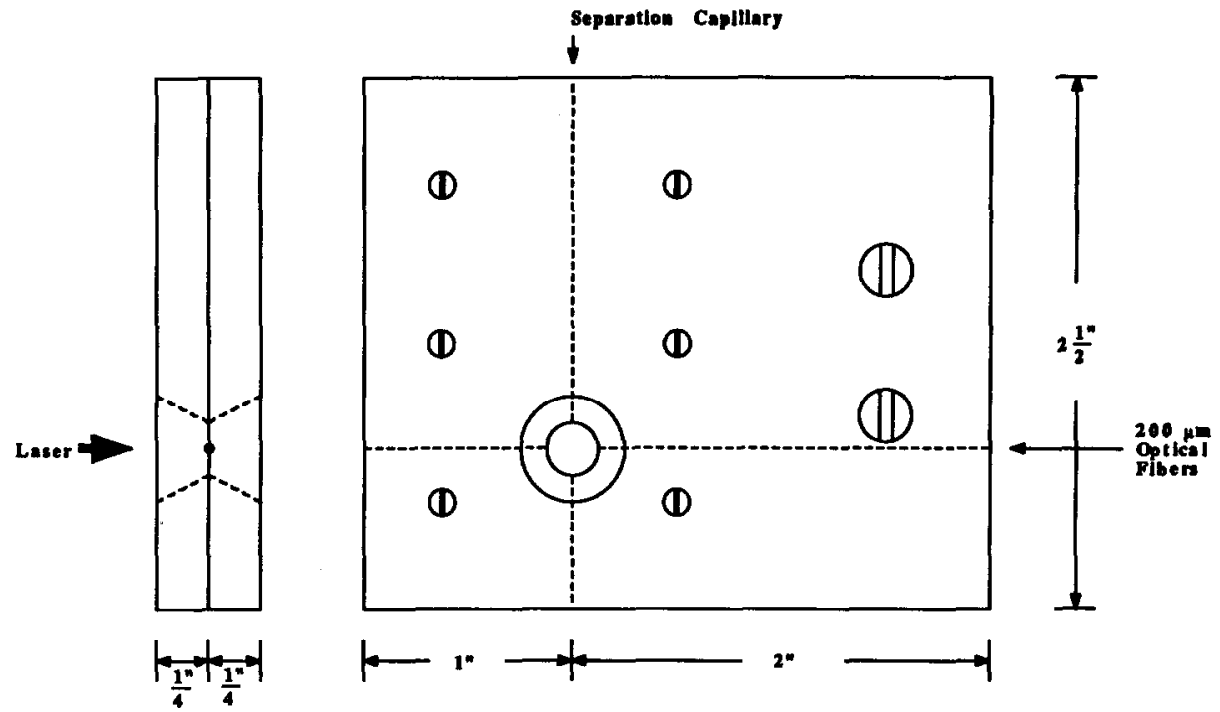

Fig. 2. Plexiglas capillary/optical fiber holder.

microscope objective (Rolyn 80.3045) focused the exciting laser beam directly on the capillary. The Raman signal was gathered by two $200-\mu \mathrm{m}$ quartz optical fibers (General Fiber Optics, 16-200S). These fibers were placed directly against the capillary and normal to the plane of polarization of the exciting laser beam. The distal ends of the fibers were oriented parallel to the spectrograph entrance slit in a Plexiglas holder. A collimating lens, $\mathrm{L}_{1},(f=50 \mathrm{~mm})$ and focusing lens $\mathrm{L}_{2},(f=200 \mathrm{~mm})$ were used to couple the fiber output into the spectrograph. The signal collected by the fibers was filtered by a sharp cut filter (Schott OG-550) to attenuate the laser line scattering.

The Raman spectrometer consisted of a $0.64-\mathrm{m}$ spectrograph (ISA Jobin Yvon HR-640) with a 1200 grooves/mm holographic grating, operated at $f / 5.7$ and a cryogenically cooled charge-coupled device detector (Photometrics series 200 , with PM 512 CCD chip). The laser was aligned to the capillary by centering the diffraction pattern obtained by focusing it into the capillary. The collection fiber alignment to the spectrograph and the spectrograph input optics were adjusted using intense fluorescence of Rhodamine 6G. For Raman spectroscopy the entrance slit was $300 \mu \mathrm{m}$, to give spectral resolution of $12 \mathrm{~cm}^{-1}$. The CCD detector chip had $512 \times 512$ pixels, each pixel $20 \mu \mathrm{m}$ square. A Raman spectrum was obtained by binning the CCD vertically to form a $512 \times 1$ data array. A personal computer with a locally written program controlled the detector, converted binary data from the CCD to ASCII arrays and stored spectra.

\section{Reagents}

The running buffer, $0.02 M$ Trizma at $\mathrm{pH} 7.15$, was prepared from Trizma base and Trizma $\mathrm{HCl}$ (Sigma). Epinephrine, phenylalanine and tryptophan were purchased from Sigma. Methyl orange and methyl red were purchased from Aldrich. All other reagents were of ACS reagents grade. All compounds were used as received. Type I water was used to prepare all solutions. 


\section{Procedure}

Adrenochrome was prepared by reaction of epinephrine with excess triiodide in the Trizma buffer. After 2-3 min, excess triiodide was destroyed by potassium thiosulfate and the adrenochrome diluted with Trizma buffer to a final concentration of $5 \cdot 10^{-4} \mathrm{M}$.

\section{RESULTS AND DISCUSSION}

The resonance enhanced Raman spectra of methyl orange and methyl red have been reported [8]. The base forms of methyl orange and methyl red have strongly enhanced $\mathrm{N}=\mathrm{N}$ stretches around $1410 \mathrm{~cm}^{-1}$. However, the absorption maxima of the basic forms of methyl orange and methyl red are 464 and $440 \mathrm{~nm}$. As shown in Fig. 3, these wavelengths are about $3000-4500 \mathrm{~cm}^{-1}$ to the blue of the frequency doubled Nd-YAG line ( $532 \mathrm{~nm}$ ). Thus, our spectra are taken at an exciting wavelength closer to pre-resonance than to true resonance excitation. Nonetheless, on-line spectra can be acquired at good signal-to-noise ratio.

The required integration time is a compromise between the signal collection needs of Raman spectroscopy and the resolution requirements of capillary electrophoresis. Fig. 4 shows a time-series of on-line Raman spectra obtained during electrophoresis of $5 \cdot 10^{-4} M$ methyl orange and methyl red in Trizma buffer at $\mathrm{pH} 7.2$. At high concentrations, $1 \cdot 10^{-4} M$ and higher, we could acquire spectra with acceptable signal-to-noise ratios with 1 or $2 \mathrm{~s}$ integration times. However, to avoid unrealistically high detection limits, we chose to increase integration times to $5 \mathrm{~s}$. Although some resolution is sacrificed by the long integration time, baseline separation of this pair of compounds is still obtained, as shown in a plot of the time-evolution of the $1416 \mathrm{~cm}^{-1}$ signal, Fig. 5. The figure also shows the presence of impurities in the sample. The peaks at 2.7 and $3.6 \mathrm{~min}$ are fluorescence, not Raman

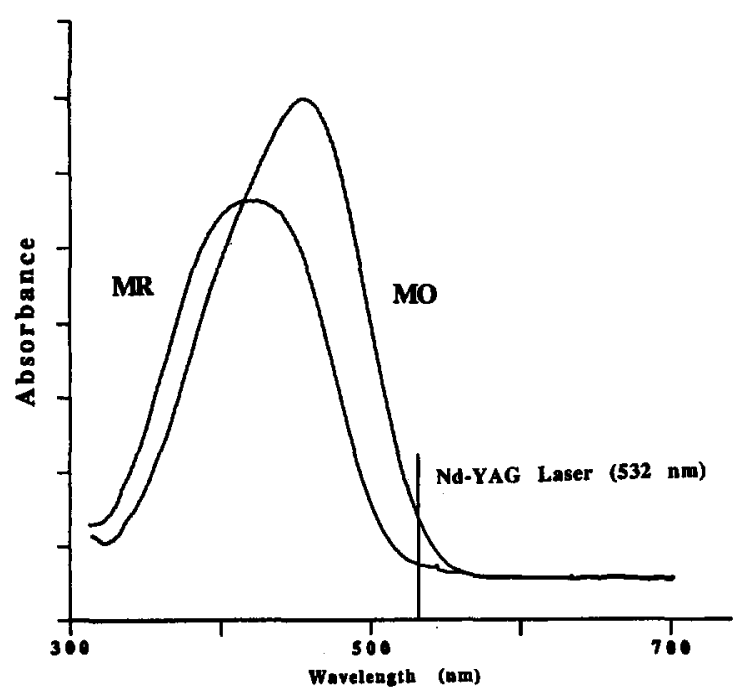

Fig. 3. Absorption spectra of methyl red (MR) and methyl orange (MO). The position of the $532 \mathrm{~nm}$ laser line is shown. 


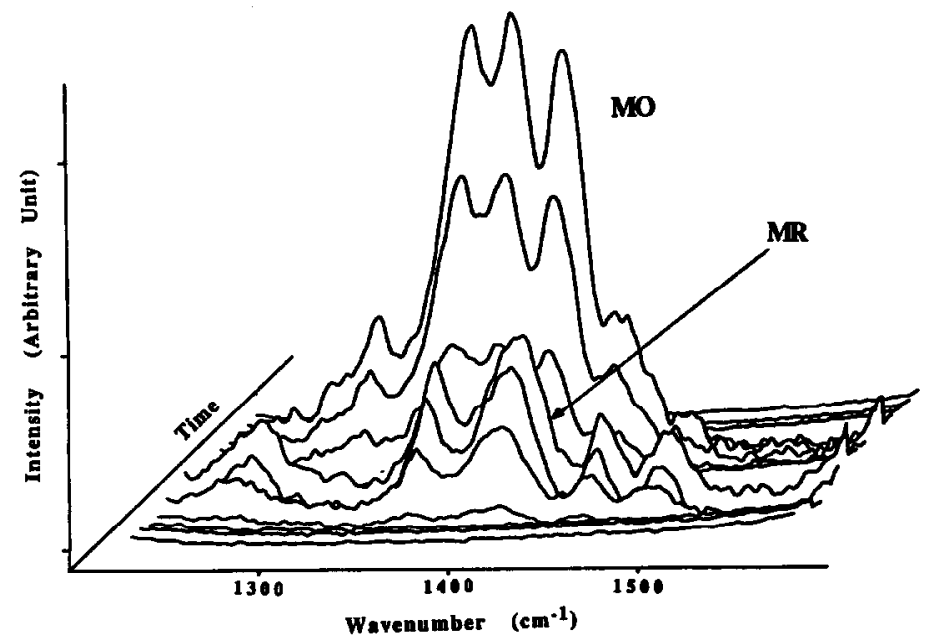

Fig. 4. Time series Raman spectra of $5 \cdot 10^{-4} \mathrm{M}$ methyl orange (MO) and methyl red (MR) in 0.02 $M$ Trizma buffer, pH 7.2. CCD integration time of $5 \mathrm{~s}$ for each spectrum. Sample injection: $20 \mathrm{kV}, 5 \mathrm{~s} ; \mathrm{CZE}$ at $20 \mathrm{kV}$; laser power: $250 \mathrm{~mW}$.

scatter. At both peaks a high background was observed, but there were no resolved Raman bands.

Despite the theoretical loss of sensitivity from the use of pre-resonance excitation, the detection limits $(\mathrm{S} / \mathrm{N}=3)$ for $5 \mathrm{~s}$ integration time, $6 \cdot 10^{-7} \mathrm{M}$, compare favorably with both absorbance detection limits and our earlier single-channel resonance Raman results [4]. That detection limits are unchanged despite the use of a less-favorable excitation wavelength can be attributed to two factors. First, the incident laser power has been increased from $40 \mathrm{~mW}$ to $250 \mathrm{~mW}$. Second, the CCD has

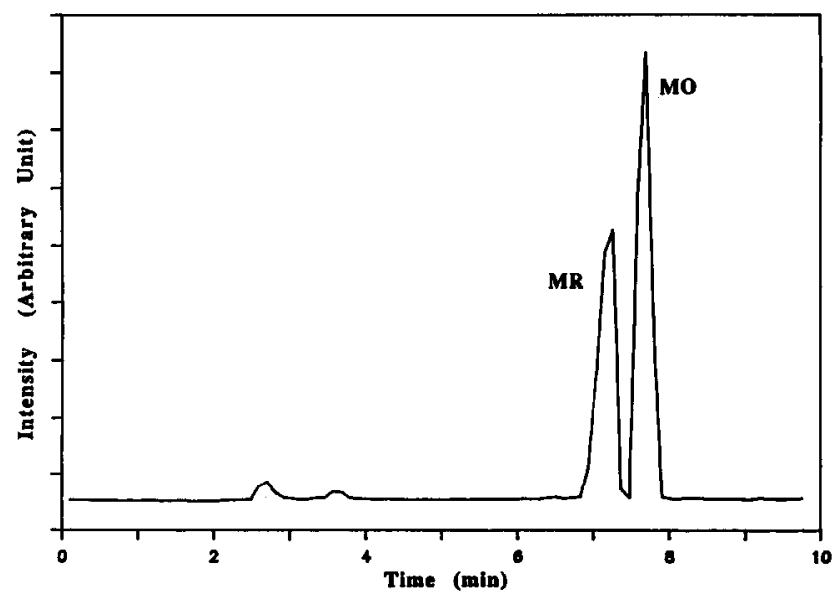

Fig. 5. Raman-detected $\left(1416 \mathrm{~cm}^{-1}\right)$ electrophoretogram of $5 \cdot 10^{-4} M$ methyl red (MR) and methyl orange (MO) in $0.02 M$ Trizma buffer. Sample injection: $20 \mathrm{kV}, 5 \mathrm{~s}$; CZE at $20 \mathrm{kV}$; laser power: $250 \mathrm{~mW}$. 
a higher quantum efficiency and is inherently quieter than the PMT employed in our earlier studies.

By increasing the integration time to $10-40 \mathrm{~s}$, we can further decrease the detection limits without increasing laser power or changing excitation wavelength. This long time scale is incompatible with sample residence time in the field of view of the detector, of course. To circumvent this problem, we reduce the driving DC voltage from $20 \mathrm{kV}$ to $7 \mathrm{kV}$ when the sample zone reaches the detection window. Fig. 6 compares the Raman spectra at the equivalent points in the electrophoretogram for a series of integration times. This strategy allows reduction of detection limits to 4 , 2 and $1 \cdot 10^{-7} M$ for 10,20 and 40 sintegration times. With $5 \mathrm{nl}$ injection volume and 40 $s$ integration time, the limit of detection (LOD) for this system is 500 attomoles $\left(5 \cdot 10^{-16}\right.$ moles).

If the width of a CZE band is strictly limited by diffusion, then there is some loss of resolution associated with reduction of the driving voltage [9]. In practice, bands are rarely as narrow as predicted from diffusion coefficients, so that the resolution loss from the increased residence time is negligible. A greater concern is the possible destruction of delicate compounds by prolonged laser irradiation. While we can not detect any decomposition of these azo compounds, driving voltage reduction is not a risk-free technique.

The most common chromatographic derivatization strategy is to attach a chromophore or fluorophore to a molecule. Typically, the accessible electronic transitions of the derivative will be localized on the added chromophore. Consequently, such a derivative will have a resonance Raman spectrum which is more characteristic of the derivatizing reagent than of the target molecule. Raman detection of such derivatives is not especially informative.

Among the simplest exceptions to this generalization are derivatives which are formed by oxidation of molecules. Although oxidation may be followed by rearrangement of the molecule, or by uptake or expulsion of small groups such as hydroxyl ions,

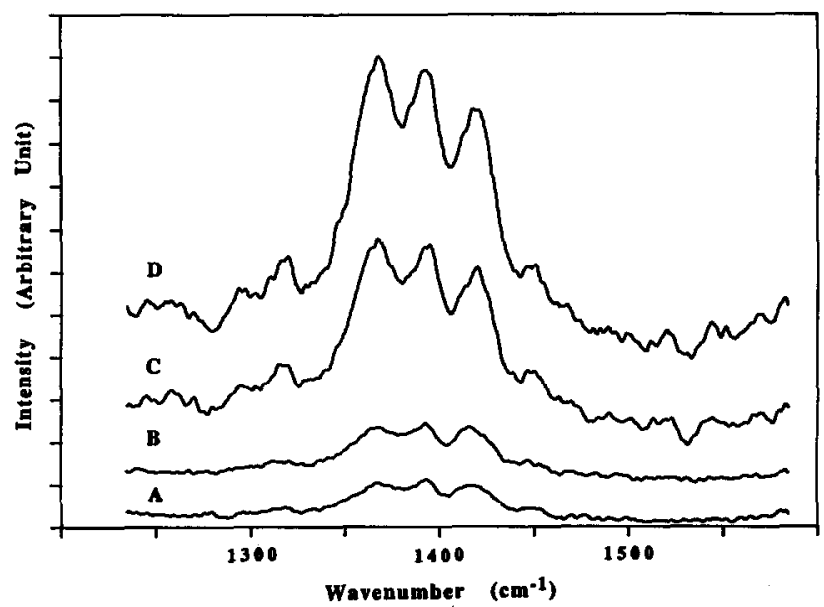

Fig. 6. On-line Raman spectra at different integration times for $5 \cdot 10^{-5} M$ methyl orange in $0.02 M$ Trizma buffer at pH 7.2. (A) $5 \mathrm{~s}$; (B) $10 \mathrm{~s}$; (C) $20 \mathrm{~s}$; (D) $40 \mathrm{~s}$. Sample injection: $20 \mathrm{kV}, 5 \mathrm{~s}$; laser power: $250 \mathrm{~mW}$. 


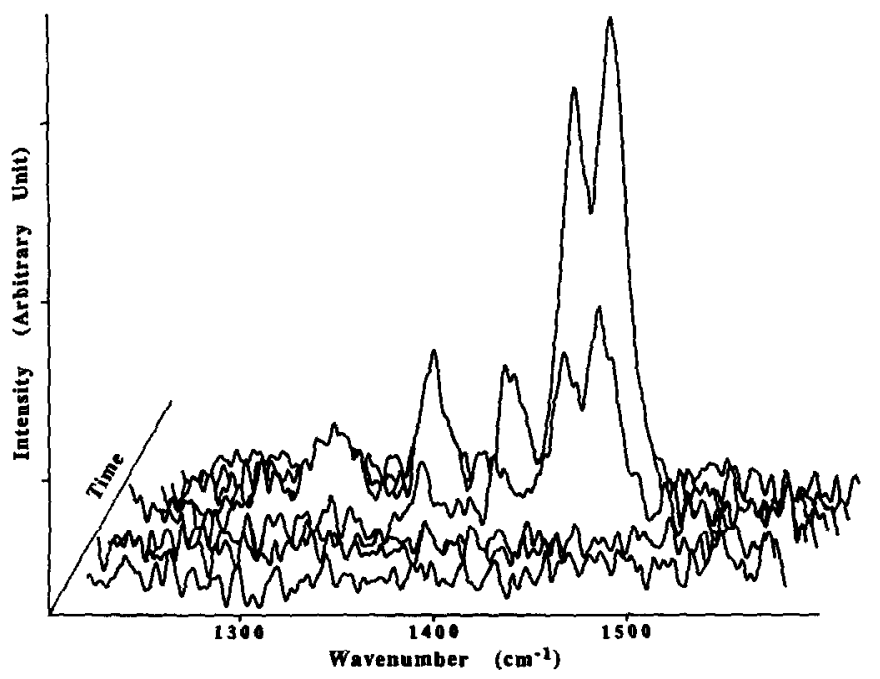

Fig. 7. Time series Raman spectra of $5 \cdot 10^{-4} M$ adrenochrome in $0.02 M$ Trizma buffer, $p H$ 7.1. CCD integration time of $20 \mathrm{~s}$ for each spectrum. Sample injection: $20 \mathrm{kV} ; 5 \mathrm{~s} ; \mathrm{CZE}$ at $20 \mathrm{kV}$; laser power $220 \mathrm{~mW}$.

the resulting final species will often reflect the structure of the starting material quite faithfully. In such cases the specificity of vibrational spectroscopy can be usefully employed. As a test of this strategy, we have chosen to oxidize epinephrine to the red-orange adrenochrome $\left(\lambda_{\max }=490 \mathrm{~nm}\right)$. Adrenochrome has relatively strong $\mathrm{C}=\mathrm{N}^{+}$or $\mathrm{C}=\mathrm{N}$ stretches at 1474 and $1454 \mathrm{~cm}^{-1}[10]$. Fig. 7 shows a time-series of on-line resonance Raman spectra of $5 \cdot 10^{-4} \mathrm{M}$ adrenochrome in Trizma buffer at $\mathrm{pH}$ 7.1. However, adrenochrome is not completely stable over time or under the high power density of our experiment. Although the samples werc filtered prior to introduction into the column, we observed flashes of scattered laser light, characteristic of suspended particles, with a longer migration time than adrenochrome itself. The light scattering is probably due to polymerization of adrenochrome to water-insoluble melanins [11]. Even so, with reduced running voltage and 20 $s$ integration, the detection limits for adrenochrome is about $10^{-5} M$. This value is about 10-fold higher than can be obtained with larger volumes in static samples.

Although it is insensitive, non-resonance Raman is not entirely unfeasible. Fig. 8 shows a time series of on-line non-resonance Raman spectra taken during electrophoretic separation of $0.05 \mathrm{M}$ of phenylalanine (PHE) and tryptophan (TRP) in Trizma buffer at $\mathrm{pH}$ 7.1. This mixture is incompletely resolved under these conditions and a non-selective detector, such as a refractive index detector, shows only a single broad peak. However, in the Raman spectrum it is easy to identify the phenylalanine. $1006 \mathrm{~cm}^{-1}$ and tryptophan $1013 \mathrm{~cm}^{-1}$ bands. Although it is just barely observable above the background noise, the $880 \mathrm{~cm}^{-1}$ band tracks tryptophan migration only. The phenylalanine $1033 \mathrm{~cm}^{-1}$ band tracks the migration of that molecule through the detector region with somewhat better signal-to-noise ratio.

Allowed in-plane aromatic ring modes are typically strong Raman bands [12]. Phenylalanine and tryptophan represent rather favorable test cases for non-resonance Raman. Comparable results would be expected for other water-soluble aromatic 


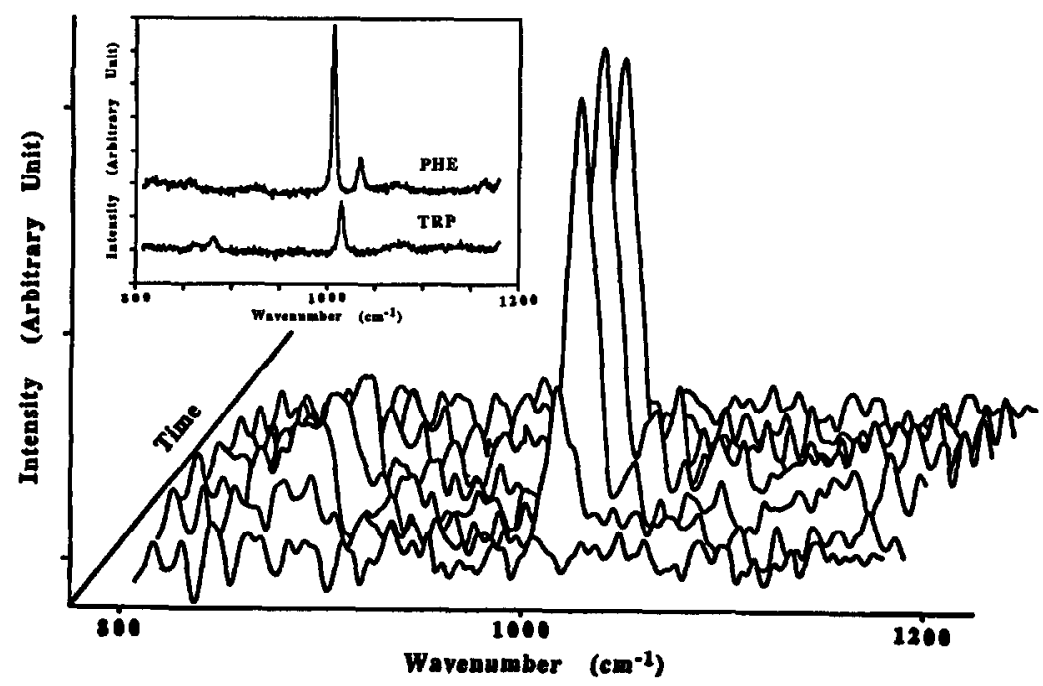

Fig. 8. Time series Raman spectra of $0.05 M$ phenylalanine and tryptophan in $0.02 M$ Trizma buffer, $\mathrm{pH}$ 7.2. CCD integration time of $10 \mathrm{~s}$ for each spectrum. Sample injection: $20 \mathrm{kV}, 10 \mathrm{~s} ; \mathrm{CZE}$ at $20 \mathrm{kV}$; laser power $200 \mathrm{~mW}$. Non-resonant Raman spectra of phenylalanine (PHE) and tryptophan (TRP) in Trizma buffer, $\mathrm{pH}$ 7.2 , is shown in inset.

molecules. Better sensitivity could be obtained by increasing laser power to several watts and by multipassing the laser beam through the sample. However, it is unlikely that detection of molecules at concentrations much below $10^{-3} M$ is feasible if the exciting wavelength is far from resonance.

\section{CONCLUSION}

The feasibility of on-line multichannel Raman spectroscopic detection for CZE has been demonstrated. However, resonance or pre-resonance enhancement is required to achieve satisfactory detection limits. To be more generally useful, excitation in the ultraviolet or near-ultraviolet is probably necessary. Because scattering intensity increases with the fourth power of frequency, required ultraviolet laser powers are quite low. The familiar $\mathrm{He}-\mathrm{Cd} 325 \mathrm{~nm}$ line may prove useful at power levels below $20 \mathrm{~mW}$, and the recently developed neon ion lasers may provide adequate power at various wavelengths between 332 and $370 \mathrm{~nm}$. In general, the use of low resolution $\left(6-12 \mathrm{~cm}^{-1}\right)$ is adequate. A wider spectral window is desirable, and could be achieved by the use of shorter focal length monochromators and coarser gratings. Our CCD is a $512 \times 512$ pixel device designed for imaging. Low noise chips optimized for spectroscopy $(1152 \times 298$ pixels $)$ are now becoming available and should also contribute to improved performance of a Raman detector.

The fiber optic collection system is quite flexible. Other fibers could be added to provide simultaneous absorbance detection slightly upstream of the Raman detector. An absorbance/Raman detector would allow optimization of the reduced velocity technique for Raman spectrum acquisition. 


\section{ACKNOWLEDGEMENTS}

This work is supported in part by NIH grant GM-37006 and by the office of Vice President for Research, the University of Michigan.

\section{REFERENCES}

1 A. G. Ewing, R. A. Wallingford and T. M. Olefirowicz, Anal. Chem., 61 (1989) 292 A.

2 M. J. Gordon, X. Huang, S. L. Pentoney, Jr. and R. N. Zare, Science (Washington, D.C.), 242 (1988) 224.

3 R. D. Smith, J. A. Olivares, N. T. Nguyen and H. R. Udseth, Anal. Chem., 60 (1988) 436.

4 C.-Y. Chen and M. D. Morris, Appl. Spectrosc., 42 (1988) 515.

5 J. V. Sweedler, R. B. Bilhorn, P. M. Epperson, G. R. Sims and M. B. Denton, Anal. Chem., 60 (1988) 282A.

6 P. M. Epperson, J. V. Sweedler, R. B. Bilhorn, G. R. Sims and M. B. Denton, Anal. Chem., 60 (1988) 327A.

7 Y.-F. Cheng, R. D. Piccard and T. Vo-Dinh, Appl. Spectrosc., 44 (1990) 755.

8 K. Machida, B.-K. Kim, Y. Saito, K. Igarashi and T. Uno, Bull. Chem. Soc. Jpn., 47 (1974) 78.

9 J. W. Jorgenson and K. D. Lukacs, Anal. Chem., 53 (1981) 1298.

10 M. D. Morris, Anal. Chem., 47 (1975) 2453.

11 M. S. Rahaman and M. D. Morris, Talanta, 23 (1976) 65.

12 F. R. Dollish, W. G. Fateley and F. F. Bentley, Characteristic Raman Frequencies of Organic Compounds, Wiley, New York, 1974. 